\title{
On the Interpretation of the Angular Dependence of the Main FMR/SWR Line in Ferromagnetic Thin Films
}

\author{
H. Puszkarski* AND M. Kasperski \\ Surface Physics Division, Faculty of Physics, Adam Mickiewicz University \\ Umultowska 85, 61-614 Poznań, Poland
}

\begin{abstract}
The reported measurements of the angular dependence of ferromagnetic resonance spectra and their theoretical interpretation lead to the observation that a discrepancy occurs between the theory and the experiment in certain ranges of the configuration angle. This disagreement cannot be eliminated within the Smit-Beljers model commonly used in the literature for the description of ferromagnetic resonance in thin films since the main ferromagnetic resonance line observed in thin films is not necessarily associated with the excitation of a uniform mode, as the Smit-Beljers model does assume for bulk samples. Instead, we associate this line with the excitation of a surface mode or the thin-film principal nonuniform mode. By taking into account the occurrence of a surface spin-pinning anisotropy, this assumption allows to obtain an agreement between the theoretical description of the ferromagnetic resonance and the experimental data in the whole range of angular configuration of the external field. As an example, on the basis of this modified ferromagnetic resonance model, we make some observations on the angular resonant dependence in uniaxial thin films.
\end{abstract}

PACS: $76.50 .+\mathrm{g}, 75.70 .-\mathrm{i}$

\section{Introduction}

Ferromagnetic resonance (FMR) in thin films is frequently studied versus the configuration of the external field with respect to the ferromagnetic film surface (see, e.g. [1-3]). The resonance spectrum can include multiple peaks or a single peak alone. In the former case it is referred to as a spin-wave resonance (SWR) spectrum, while a single-peak spectrum is regarded as a classic ferromagnetic resonance one. In this paper we discuss the configurational dependence of the resonance field of the single FMR line or, in the case of SWR, the first main line (of the sequence of resonance lines ordered with decreasing resonance fields). Experimentalists tend to use the classic Smit-Beljers (S-B) resonance formula $[4,5]$ for the theoretical description of the dependence of the resonance field corresponding to this main FMR line on the angle $\theta_{H}$ between the film normal and the external field. However, very often the $H_{\mathrm{res}}^{\exp }\left(\theta_{H}\right)$ dependence found in the experiment proves to differ from the theoretical dependence $H_{\mathrm{res}}^{\mathrm{S}-\mathrm{B}}\left(\theta_{H}\right)$ calculated in the Smit-Beljers model in certain ranges of $\theta_{H}$. In this study we discuss the possible causes of this interpretational discrepancy.

The first observation to be made in our considerations is that the $\mathrm{S}-\mathrm{B}$ formula was originally derived for bulk materials, large enough to neglect the effect of boundary conditions and therefore assume that a so-called uniform mode, with wave vector $\boldsymbol{k}\left[k_{x}, k_{y}, k_{z}\right] \equiv 0$, is excited in the FMR. However, this assumption cannot be made without reservations in the case of thin films. Even when the film is very large in the directions parallel to its surface we can at most assume $\boldsymbol{k}_{\|}\left[k_{x}, k_{y}\right] \equiv 0$; a similar assumption

\footnotetext{
* corresponding author; e-mail: henpusz@amu.edu.pl
}

for the wave-vector component perpendicular to the film would be unjustified. We must assume that in general $k_{\perp} \neq 0$ in thin films.

It is a well-known fact that the boundary conditions implied by the occurrence of the two surfaces in a thin film determine the allowed sequence of $k_{\perp}$ values associated with the respective standing spin waves that form in the direction perpendicular to the film. In this sequence, the wave number $k_{\perp}$ corresponding to the principal harmonic - or the fundamental mode, corresponding to the strongest resonance field - is not necessarily identical with zero; in general we must assume that, due to the boundary conditions, $k_{\perp}^{\text {fund }} \neq 0$. We argue that the condition $k_{\perp}^{\text {fund }} \approx 0$ is only fulfilled in those ranges of the angle $\theta_{H}$ in which the experimental resonance fields correspond to the $\mathrm{S}-\mathrm{B}$ resonance curve; in the other regions, in which a discrepancy occurs between the theoretical and experimental results, we allow for a nonuniform resonance mode with $k_{\perp}^{\text {fund }}$ substantially different from zero. The additional exchange energy related to the excitation of this nonuniform mode with $k_{\perp}^{\text {fund }} \neq 0$ is the very cause of the discrepancy between the resonance field $H_{\text {res }}^{\text {fund }}\left(\theta_{H}\right)$ found experimentally and the value calculated by the $\mathrm{S}-\mathrm{B}$ formula derived on the assumption that $k_{\perp}^{\text {fund }} \equiv 0$. This idea will be set out below in detailed considerations based on the quantum theory of SWR we have developed and presented in our previous publications [6-9].

\section{Application of Smit-Beljers resonance formula to thin films}

Let us consider the simplest example of ferromagnetic thin film with uniaxial anisotropy, discussed recently e.g. by Beaujour et al. [10, 11]. We shall regard the free energy $E$ of the sample as the sum of the Zeeman, demagnetization and uniaxial anisotropy energies 


$$
E=-\boldsymbol{M} \cdot \boldsymbol{H}+2 \pi(\boldsymbol{M} \cdot \boldsymbol{n})^{2}-K(\boldsymbol{M} \cdot \boldsymbol{u} / M)^{2},
$$

where $\boldsymbol{M}$ is the magnetization, $\boldsymbol{H}$ is the applied magnetic field, $\boldsymbol{n}$ denotes a unit vector in the direction normal to the film surface, $\boldsymbol{u}$ is a unit vector in the direction of the easy-magnetization axis, and $K$ denotes the effective uniaxial anisotropy constant. We shall henceforth assume $\boldsymbol{u}=\boldsymbol{n}$; thus, the free energy can be expressed as

$$
\begin{aligned}
E & =-M H\left(\sin \theta \sin \theta_{H}+\cos \theta \cos \theta_{H}\right) \\
& +\left(2 \pi M^{2}-K\right) \cos ^{2} \theta .
\end{aligned}
$$

In the derivation of the above formula we have assumed that the film surface defines the $(x, y)$ plane; $z$ is the direction normal to this plane; $\theta$ and $\theta_{H}$ are the angles between the magnetization $\boldsymbol{M}$ or the external field $\boldsymbol{H}$, respectively, and the normal to the film surface; both $\boldsymbol{M}$ and $\boldsymbol{H}$ are assumed to lie in the $(z, y)$ plane. The SmitBeljers resonance formula reads $[4,12]$ :

$$
\left(\frac{\omega}{\gamma}\right)^{2}=\frac{1}{M^{2} \sin ^{2} \theta}\left[\frac{\partial^{2} E}{\partial \phi^{2}} \frac{\partial^{2} E}{\partial \theta^{2}}-\left(\frac{\partial^{2} E}{\partial \phi \partial \theta}\right)^{2}\right],
$$

where $\omega$ is the angular resonance frequency, and $\gamma$ $\left(=g \mu_{\mathrm{B}} / \hbar\right)$ is the gyromagnetic ratio. The following configurational resonance condition (that allows also for the perpendicular configuration of the external field, $\theta_{H} \equiv$ $\theta=0)$ results from (2) and (3):

$$
\begin{aligned}
& \left(\frac{\omega}{\gamma}\right)^{2}=\left[H \cos \left(\theta-\theta_{H}\right)-(4 \pi M-2 K / M) \cos 2 \theta\right] \\
& \quad \times\left[H \cos \left(\theta-\theta_{H}\right)-(4 \pi M-2 K / M) \cos ^{2} \theta\right] ;
\end{aligned}
$$

for a given direction $\theta_{H}$ of the applied magnetic field the equilibrium position $\theta$ of the magnetization is calculated from the condition $\partial E / \partial \theta=0$ and is given by the relation

$$
2 H_{\text {res }} \sin \left(\theta-\theta_{H}\right)=(4 \pi M-2 K / M) \sin 2 \theta .
$$

Let us introduce, for convenience, a quantity referred to as effective demagnetization field $M_{\text {eff }}$, defined $[10,11]$ as:

$$
4 \pi M_{\mathrm{eff}}=4 \pi M-2 K / M .
$$

To realize well the limitations to be taken into consideration when the Smit-Beljers resonance formula (4) is used in the theory of thin films, let us refer now to the microscopic theory of ferromagnetic resonance in thin films. The pertinent case of thin film with a "perpendicular" uniaxial anisotropy is considered within the microscopic model in our earlier papers [9, 13], which we shall now refer to. In these papers the uniaxial anisotropy energy is expressed by the following single-ion term of the Hamiltonian:

$$
\hat{H}_{\text {anis }}=-D(l) \sum_{l j}\left(\hat{S}_{l j}^{z}\right)^{2},
$$

where $l j$ defines the position of the given spin: $l$ labels the atomic plane (parallel to the film surface) and $j$ is a two-dimensional vector defining the position of the spin in the $l$-th plane; $D(l)$ denotes the single-ion uniaxial anisotropy constant for a spin in the $l$-th atomic plane. Now, the resonance condition obtained in [9] [see
Eq. (5.7)] can be expressed by the material quantities defined at the macroscopic level and introduced above; we shall use as the basis the following identity relations between the microscopic and macroscopic quantities for the effective demagnetization field and the exchange constant, respectively

$$
4 \pi M_{\mathrm{eff}}=-\frac{2 D S}{g \mu_{\mathrm{B}}}, \quad D_{\mathrm{ex}}=\frac{2 S z_{\perp} J a^{2}}{g \mu_{\mathrm{B}}} .
$$

The meaning of the microscopic quantities (at the right side of the above equalities) introduced in [9] is the following: $D$ is the uniaxial (single-ion) anisotropy constant, $S$ denotes the spin (in $\hbar$ units), $J$ is the nearest-neighbor exchange integral, $2 z_{\perp}$ is the number of nearest neighbors lying in adjacent atomic planes, and $a$ denotes the lattice constant. Expressed by the macroscopic quantities introduced above, the resonance condition obtained within the microscopic model in [9] becomes now

$$
\begin{aligned}
& \left(\frac{\omega}{\gamma}\right)^{2}=\left[H \cos \left(\theta-\theta_{H}\right)-4 \pi M_{\text {eff }} \cos 2 \theta+D_{\text {ex }} k_{\perp}^{2}\right] \\
& \quad \times\left[H \cos \left(\theta-\theta_{H}\right)-4 \pi M_{\text {eff }} \cos ^{2} \theta+D_{\text {ex }} k_{\perp}^{2}\right],
\end{aligned}
$$

where $D_{\text {ex }}$ is the exchange constant, and $k_{\perp}$ denotes the wave number of the standing spin wave. Note that Eq. (9) is known in the literature for years (cf., e.g. [1, 14]); however, its derivation here has led us to formulate the identity relations (8), which shall be of much use in our further considerations. The resonance condition (9) corresponds to the excitation of a standing spin wave with wave number $k_{\perp}$; only for the excitation of a uniform mode $\left(k_{\perp}=0\right)$ does this condition reduce to the SmitBeljers formula (4). However, it should be stressed that $k_{\perp}=0$ only in a special case that corresponds to a thin film without any surface anisotropy! This means that reversing the direction of our reasoning - if we want to use the Smit-Beljers formula for the description of the main resonance line in a thin-film spectrum, we must allow for the fact that in general this line can correspond to the excitation of a nonuniform fundamental mode (with a nonzero wave number, $k_{\text {fund }} \neq 0$ ); therefore, an extra energy term, corresponding to the energy of excitation of this mode, must be added in the $\mathrm{S}-\mathrm{B}$ resonance condition

$$
\begin{aligned}
& \left(\frac{\omega}{\gamma}\right)^{2}= \\
& {\left[H \cos \left(\theta-\theta_{H}\right)-4 \pi M_{\mathrm{eff}} \cos 2 \theta+D_{\mathrm{ex}}\left(k_{\perp}^{\text {fund }}\right)^{2}\right]} \\
& \quad \times\left[H \cos \left(\theta-\theta_{H}\right)-4 \pi M_{\mathrm{eff}} \cos ^{2} \theta+D_{\mathrm{ex}}\left(k_{\perp}^{\text {fund }}\right)^{2}\right] .
\end{aligned}
$$

The additional exchange energy term introduced above, $+D_{\text {ex }} k_{\text {fund }}^{2}$, changes the angular dependence of the resonance field $H_{\text {res }}=H_{\text {res }}\left(\theta_{H}\right)$ resulting solely from the condition (4), since in general the boundary conditions impose also the angular dependence $k_{\text {fund }}=k_{\text {fund }}(\theta)$ of the fundamental mode. The configurational dependence of the fundamental mode in uniaxial thin films is discussed in detail in the next paragraph. 


\section{Configurational dependence of the thin-film fundamental mode}

The value of the wave number $k_{\perp}^{\text {fund }}$ of the fundamental mode in a thin film is determined by the boundary conditions on the film surfaces. In the microscopic theory of spin waves the boundary conditions are described by the surface pinning parameter, which measures the degree (strength) of "pinning" of the surface spins. (A correlated "pinning" quantity is the surface anisotropy energy, used in the macroscopic description of spin waves in thin films.) If the thin film under consideration meets the assumptions of the surface inhomogeneity (SI) model [6-9] the formula for the surface parameter can be derived in a way analogical to that presented in our earlier paper [13]. In the case considered here the result is

$$
A=1-\frac{D_{\mathrm{b}}-D_{\mathrm{s}}}{2 z_{\perp} J}\left(1-3 \cos ^{2} \theta\right),
$$

where $D_{\mathrm{b}}$ and $D_{\mathrm{s}}$ denote the bulk and surface microscopic uniaxial anisotropy constants, respectively. Now, using the identity relations (8) we get the formula in which the surface parameter is expressed by macroscopic quantities

$$
A(\theta)=1-\frac{1}{2}\left[4 \pi\left(M_{\mathrm{eff}}^{\mathrm{surf}}-M_{\mathrm{eff}}^{\mathrm{bulk}}\right) \frac{a^{2}}{D_{\mathrm{ex}}}\right]\left(1-3 \cos ^{2} \theta\right) ;
$$

the effective demagnetization fields $M_{\text {eff }}^{\text {surf }}$ and $M_{\text {eff }}^{\text {bulk }}$ in (12) are as defined in (6) with substituted surface or bulk quantities, $M^{\text {surf }}$ and $K^{\text {surf }}$ or $M^{\text {bulk }}$ and $K^{\text {bulk }}$, respectively. The surface parameter $A$ proves to depend on $\theta$ when the effective demagnetization fields at the surface and in the bulk are different, i.e.

$$
\Delta M_{\mathrm{eff}} \equiv M_{\mathrm{eff}}^{\text {surf }}-M_{\mathrm{eff}}^{\mathrm{bulk}} \neq 0 .
$$

If the condition (13) is fulfilled the surface parameter $A$ varies with the angle $\theta$ by oscillating around $A=1$, the value corresponding to a certain angle - let us refer to it as critical angle - for which $\left(1-3 \cos ^{2} \theta\right) \equiv 0$.

Figure 1 (based on our earlier papers [6-9]) presents the profiles of the fundamental mode corresponding to different values of $A$. The fundamental mode is seen to be a uniform mode only for the particular surface parameter value $A=1$ (which implies the lack of surface anisotropy) corresponding to the critical angle; for $A<1$ the fundamental mode is a principal nonuniform bulk mode, and becomes a surface mode for $A>1$. Thus, the application of the S-B formula to thin films is only justified for the external field configuration corresponding to the critical angle; for any other angle the formula does not apply! For this very reason the resonance curve $H_{\text {res }}=H_{\text {res }}\left(\theta_{H}\right)$ found experimentally differs (in some cases) from the theoretical curve based on the Smit-Beljers model (see, e.g., $[1,2])$.

\section{Conclusion}

In the present paper we argue that the Smit-Beljers resonance formula can only be applied to thin films without surface anisotropy, i.e. with surface spins pinned to

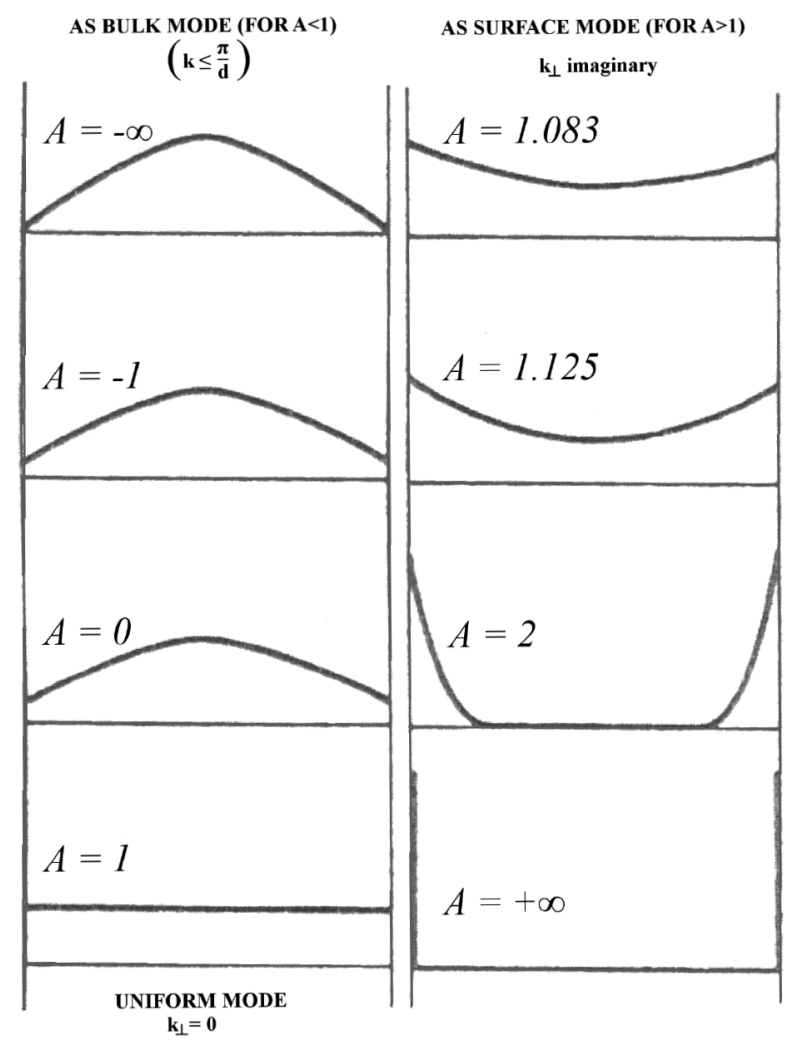

Fig. 1. Profiles of the thin-film fundamental standing spin-wave mode given by the surface inhomogeneity model for different values of surface pinning parameter $A$. Only for a well-defined value of $A=1$ the fundamental mode has the uniform profile corresponding to a zero wave number $k_{\perp}$ of the component perpendicular to the film surface. For $A>1$ the fundamental mode is surface-localized, and for $A<1$ the principal bulk spin-wave mode in the thin film resembles the first harmonic mode of string vibration $(d=10 a$ denotes the film thickness).

the extent corresponding to their natural freedom only resulting from the presence of the surface. Only in this case the fundamental mode excited in ferromagnetic resonance is the uniform mode for which the $\mathrm{S}-\mathrm{B}$ formula was derived. In the case of additional surface spin pinning due to the surface anisotropy the fundamental mode is not uniform, and the application of the classical S-B formula to the description of FMR in thin films is unjustified. To become applicable, the formula must be completed by an extra energy term, $+D_{\text {ex }} k_{\text {fund }}^{2}$, corresponding to the exchange energy of excitation of the nonuniform $\left(k_{\text {fund }} \neq 0\right)$ fundamental mode (see (10)).

We have also demonstrated that in the case of uniaxial surface anisotropy the nature of the fundamental mode in the thin film changes with the configuration of the external magnetic field with respect to the film surface: it is a bulk mode in some ranges of the configuration angle, but in other ranges - the fundamental mode is of surface localized character. This finding allows to hope 
that the disagreement observed between the experimental and theoretical (based on the classical S-B formula) configurational dependences of the resonance field of the main FMR line will be eliminated if the main resonance mode is assumed to be in general nonuniform. Moreover, an appropriate fitting procedure when used for the elimination of this discrepancy can yield precious information on the nature of the surface anisotropy. We shall discuss this problem in another paper.

\section{Acknowledgments}

This study was supported by the Polish Ministry of Science and Higher Education, grant No. N N202 194533.

\section{References}

[1] J.T. Yu, R.A. Turk, P.E. Wigen, Phys. Rev. B 11, 420 (1975).

[2] J. Dubowik, F. Stobiecki, I. Gościańska, M. Kociemba, W. Bednarski, Acta Phys. Pol. A 115, 315 (2009).

[3] X. Liu, Y.Y. Zhou, J.K. Furdyna, Phys. Rev. B 75, 195220 (2007).

[4] J. Smit, H.G. Beljers, Philips Res. Rep. 10, 113 (1955).
[5] H. Suhl, Phys. Rev. 97, 555 (1955).

[6] H. Puszkarski, Acta Phys. Pol. A 38, 217 and 899 (1970).

[7] H. Puszkarski, Prog. Surf. Sci. 9, 191 (1979).

[8] H. Puszkarski, "Spin Wave Resonance - A Tool For the Study of Magnetic Surface Interactions", Ch. 9 in Proc. Twenty Second Scottish Universities Summer School on Physics, Magnetism in Solids - Some Current Topics, Eds. A.P. Cracknell. R.A. Vaughan, University of Dundee, Edinburgh1981.

[9] H. Puszkarski, Phys. Status Solidi B 96, 61 (1979).

[10] J.-M.L. Beaujour, W. Chen, K. Krycka, C.-C. Kao, J.Z. Sun, A.D. Kent, Eur. Phys. J. B 59, 475 (2007).

[11] J.-M.L. Beaujour, A.D. Kent, D. Ravelosona, I. Tudosa, E.E. Fullerton, J. Appl. Phys. 109, 033917 (2011).

[12] L. Baselgia, M. Warden, F. Waldner, S.L. Hutton, J.E. Drumheller, Y.Q. He, P.E. Wigen, M. Maryśko, Phys. Rev. B 38, 2237 (1988).

[13] Diep-The-Hung, H. Puszkarski, J.C.S. Lévy, Le Vide, les Couches Minces 204, 313 (1980).

[14] R.F. Soohoo, Magnetic Thin Films, Harper and Row, New York 1965 